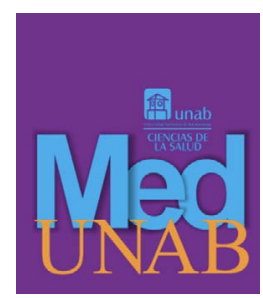

REVISTA DE LA FACULTAD

DE CIENCIAS DE LA SALUD

\title{
Morbilidad neonatal extrema y sus desenlaces en un hospital de alta complejidad en el 2013
}

Extreme neonatal morbidity and its outcomes in a high complexity hospital in 2013

Morbidade neonatal extrema e seus resultados em um hospital de alta complexidade em 2013

Angélica María Delgado-Beltrán, MD, Esp. ${ }^{1}$ (D), Mónica Andrea Beltrán-Avendaño, $M D$, Esp. $^{2}$ iD, Luis Alfonso Pérez-Vera, MD, Esp. ${ }^{3}$ (D)

1. Médica, Universidad Autónoma de Bucaramanga. Especialista en Pediatría, Universidad Industrial de Santander. Pediatra del Servicio de Urgencias y Calidad Hospital Internacional de Colombia, Servicio de Hemato-Oncología Pediátrica Hospital Universitario de Santander. Docente Cátedra de Pediatría Universidad Industrial de Santander. Bucaramanga, Santander, Colombia.

2. Médica. Especialista en Ginecología y Obstetricia, Universidad Industrial de Santander. Especialista en Medicina Materno Fetal Universidad del Rosario. Fellow en Ecocardiografía y Neurosonografía Universidad de Concepción Chile. Docente titular de Medicina Universidad Autónoma de Bucaramanga. Docente titular de cátedra Universidad Industrial de Santander. Bucaramanga, Santander, Colombia.

3. Médico. Especialista en Pediatría Universidad de Antioquia. Neonatología Universidad Militar Nueva Granada. Profesor titular del departamento de Pediatría Universidad Industrial de Santander. Bucaramanga, Santander, Colombia.

Correspondencia. Angélica María Delgado-Beltrán. Carrera 23 \# 52 - 52 apartamento 705 torre B Torres de Mardelia. Email. angy624@gmail.com

\section{INFORMACIÓN DEL ARTÍCULO:}

Artículo recibido: 03 de agosto de 2020

Artículo aceptado: 05 de marzo de 2021

DOI: https://doi.org/10.29375/01237047.3960

Cómo citar.Delgado-Beltrán AM., Beltrán-Avendaño MA., Pérez-Vera LA. Morbilidad neonatal extrema y sus desenlaces en un hospital de alta complejidad en el 2013. MedUNAB. 2021;24(1): 61-71. Doi: https://doi.org/10.29375/01237047.3960

\section{RESUMEN}

Introducción. La Morbilidad neonatal extrema se refiere a cualquier evento ocurrido en los primeros veintiocho días de vida con riesgo vital inminente. Es multifactorial, son relevantes los retrasos en la atención. En 2010 se describió por primera vez como indicador de salud pública en Brasil. Metodología. Estudio transversal en un 
hospital de alta complejidad en el año 2013. Se revisaron las características sociodemográficas y asistenciales, los eventos de morbilidad y sus desenlaces, el indicador mismo, la mortalidad neonatal y los retrasos en la atención (tipos I - IV). Resultados. Ingresaron 1,190 neonatos, se presentaron 120 casos y se analizaron los primeros 60 por saturación de datos. La tasa de morbilidad neonatal extrema fue de 59 por cada 1,000 nacidos vivos y la de mortalidad de 13.9, similares a las brasileñas y superiores a las nacionales (33 por cada 1,000). Los eventos más importantes con respecto a mortalidad fueron peso menor de 1,500 gramos, ventilación mecánica, Apgar menor de 7 a los 5 minutos y malformaciones congénitas. Los retrasos en las madres fueron predominantemente de tipo I (45.4\%) por pobre control prenatal. El retraso neonatal más frecuente fue el tipo IV (40\%), por mala adherencia a protocolos institucionales. Discusión. El indicador local fue mayor que el nacional por la complejidad institucional y los retrasos en la atención, los cuales causaron saturación estadística. Se puede mejorar con mayor adherencia a estrategias existentes como el control prenatal y protocolos institucionales. Conclusión. La Morbilidad neonatal extrema visibiliza las falencias en atención materno - neonatal.

Palabras Claves:

Recién nacido; Potencial evento adverso en cuidado de la salud; Muerte perinatal; Mortalidad; Calidad de la atención de salud.

\section{ABSTRACT}

Introduction. Extreme neonatal morbidity refers to any event that occurs in the first twenty-eight days of life with imminent vital risk. It is multifactorial and delays in treatment are relevant. It was described for the first time as a public health indicator in Brazil in 2010. Methodology. This was a cross-sectional study in a high complexity hospital in 2013. Sociodemographic and assistance characteristics, morbidity events and their outcomes, the indicator itself, neonatal mortality, and delays in treatment (types I - IV) were reviewed. Results. 1,190 neonates were entered, 120 cases occurred and the first 60 were analyzed due to data saturation. The extreme neonatal morbidity rate was 59 for every 1,000 children born alive and the mortality rate was 13.9. This was similar to the figures in Brazil and greater than national statistics (33 for every 1,000). The most significant events with respect to mortality were weight lower than 1,500 grams, mechanical ventilation, an Apgar score lower than 7 at 5 minutes and congenital anomalies. Delays from mothers were predominantly type I (45.4\%) due to poor prenatal control. The most frequent neonatal delay was type IV $(40 \%)$ due to bad adherence to institutional protocols. Discussion. The local indicator was greater than the national indicator due to the institutional complexity and delays in treatment, which cause statistical saturation. This can be improved with better adherence to existing strategies, such as prenatal control and institutional protocols. Conclusion. Extreme neonatal morbidity shows the flaws in maternal - neonatal healthcare.

Keywords:

Newborn; Potential Adverse Healthcare Event; Perinatal Mortality; Mortality; Healthcare Quality.

\section{RESUMO}

Introdução. A morbidade neonatal extrema refere-se a qualquer evento que ocorra nos primeiros vinte e oito dias de vida com risco de vida iminente. É multifatorial, e os atrasos no atendimento são relevantes. Em 2010 foi descrita pela primeira vez como um indicador de saúde pública no Brasil. Metodologia. Estudo transversal em um hospital de alta complexidade em 2013. Foram revisadas as características sociodemográficas e assistenciais, os eventos de morbidade e seus resultados, o próprio indicador, a mortalidade neonatal e os atrasos no atendimento (tipos I - IV).

Resultados. Foram internados 1,190 recém-nascidos, foram identificados 120 casos e os primeiros 60 foram analisados devido à saturação dos dados. A taxa de morbidade neonatal extrema foi de 59 por 1,000 nascidos vivos e a taxa de mortalidade de 13,9, semelhante às taxas brasileiras e superior às taxas nacionais $(33$ por 1,000$)$. Os eventos mais importantes em relação à mortalidade foram peso inferior a 1,500 gramas, ventilação mecânica, Apgar menor que 7 em 5 minutos e malformações congênitas. Os atrasos nas mães foram predominantemente do tipo I (45.4\%) 
devido ao cuidado pré-natal precário. O atraso neonatal mais frequente foi do tipo IV (40\%), devido à falta de aderência aos protocolos institucionais. Discussão. $\mathrm{O}$ indicador local foi superior ao nacional devido à complexidade institucional e aos atrasos no atendimento, o que causou saturação estatística. Pode ser melhorado com uma maior adesão às estratégias existentes, como atendimento pré-natal e protocolos institucionais. Conclusão. A morbidade neonatal extrema torna visíveis as deficiências no cuidado materno-neonatal.

Palavras-chave:

Recém-nascido; Potencial evento adverso no cuidado à saúde; Morte perinatal; Mortalidade; Qualidade da assistência à saúde.

\section{Introducción}

En Colombia, como una propuesta nueva de vigilancia epidemiológica (diseñada en 2013 y publicada en 2014), se definió la morbilidad neonatal extrema (MNE) a cualquier evento ocurrido desde el nacimiento hasta el día veintiocho de vida que haya ocasionado riesgo vital inminente; su presencia está ligada a factores sociodemográficos y asistenciales, lo que incluye la atención de la materna, el entorno social, la comunidad y la economía (1).

La literatura científica que soporta el uso de este indicador data desde 2010, cuya propuesta fue hecha operativamente en Brasil por la Dra. Pileggi (2); sin embargo, el concepto existe desde el 2007, derivado de los esfuerzos por reducir la mortalidad neonatal $(3,4)$. Se ha propuesto restringir la observación a los eventos dentro de la primera semana de vida, pero se ha mantenido la definición inicial por ser más inclusiva. El concepto actual se delimitó en el 2015 por el mismo grupo de la Dra. Pileggi, e incluyó tres criterios clínicos (Apgar $<7$ al $5^{\circ}$ minuto, peso al nacer $<1,750 \mathrm{~g} \mathrm{y}$ edad gestacional $<33$ semanas) y doce de manejo (antibióticos, CPAP nasal, intubación en primeros 7 días, fototerapia en primeras 24 horas, reanimación, vasoactivos, anticonvulsivantes, surfactantes, transfusiones, corticosteroides por hipoglucemia, cirugías), pudiendo presentarse cualquiera de estos marcadores. La tasa de MNE reportada con estos criterios es de 21.4 por cada 1,000 nacidos vivos. Es preciso mencionar que los estudios que incluyen este criterio combinado reportan un índice de mortalidad menor (10.5\%), aunque detectan más casos en riesgo; también debe tenerse en cuenta que ninguna estrategia detecta todas las muertes neonatales, siendo un $93 \%$ el máximo de detección (5-8).

En la literatura médica colombiana, al momento de diseñar este estudio en el 2013, sólo se habían realizado algunos estudios sobre factores de riesgo para mortalidad neonatal, donde los siguientes factores se asociaron con las muertes: procedencia de área rural, controles prenatales, peso al nacer menor a 2,500 g, Apgar al minuto, requerimiento de reanimación cardiopulmonar y atención inicial en un centro de baja complejidad (9-12). Todos estos factores están contemplados en la definición de la MNE.

En el 2013 era relevante hablar de MNE, puesto que en Colombia la mortalidad en menores de 5 años se había reducido casi a la mitad en los últimos 30 años, pero sin reducir suficientemente la mortalidad neonatal que contribuía aún con cerca del $50 \%$ de los casos. Como dato favorable, las mismas estadísticas mostraban que la mortalidad neonatal colombiana (temprana y tardía) frente a la de menores de 5 años era inferior a la global: se reportaron 5.2 y 2.2 vs 17.9 muertes por cada 1,000 nacidos vivos en Colombia, respectivamente; en comparación, se observaron 14 y 4.4 vs 44 muertes por cada 1000 nacidos vivos en el mundo (13). En contraste, se conocía que la tasa de mortalidad perinatal era de 16.4 muertes por cada 1,000 nacidos vivos en el 2012, con las muertes fetales constituyendo el $50 \%$ de dichos reportes (14).

En el modelo de vigilancia de MNE, dentro del análisis de causalidad de los casos, se incluye el modelo de los tres retrasos desarrollado en 1994 (buscar ayuda, alcanzar la ayuda y recibir ayuda adecuada); esta categorización de factores fue incorporada en el análisis de morbilidad neonatal extrema propuesto en nuestro país, con una subdivisión del retraso tipo III en dos retrasos (referencia a un servicio de calidad y eventos que determinan la atención de salud en sí misma). El análisis de los retrasos en la atención permite incorporar factores sociales, económicos y burocráticos dentro de la metodología, siendo una de las propuestas más nuevas $(1,15)$.

La presentación de la metodología en Colombia incluyó una prueba piloto realizada en las instituciones participantes entre febrero y marzo de 2013, incluyendo criterios operativos adicionales a la definición internacional de MNE como cardiopatías congénitas y malformaciones mayores que ameritan intervenciones, 
criterios de falla orgánica, diálisis, antiarrítmicos y cardioversión. Se analizaron datos de 89 neonatos en 6 ciudades principales, con el $71.9 \%$ de los casos agrupados en cuatro instituciones. Como hallazgos principales se obtuvo una tasa de MNE de 33 por cada 1,000 nacidos vivos; se encontró que las principales causas de MNE fueron: prematurez, infecciones, asfixia perinatal, malformaciones congénitas y enfermedades respiratorias; los retrasos más frecuentes fueron tipo IV, relacionados con fallas en atención materna (1). La mayor MNE observada fue atribuida a estudiar una población de referencia (III y IV nivel de atención) y a los retrasos en la atención $(6,16)$.

En la actualidad colombiana esto es un tema vigente, dado que el Plan Decenal de Salud Pública 2012 - 2021 contempla la reducción de la mortalidad perinatal, y el país aún está por debajo de las metas $(17,18)$; en consecuencia, se hace necesario mejorar la caracterización de los eventos de mortalidad y precisar mejor cuáles son los neonatos de mayor riesgo, quienes son identificables a través de la MNE. En este contexto, los retrasos de la atención materna se han reportado plenamente asociados con este indicador $(19,20)$.

Basado en lo anterior, el objetivo del presente estudio fue realizar la primera caracterización colombiana de eventos de MNE, incluyendo, como aspecto novedoso, el análisis de los retrasos en la atención (maternos y neonatales) que posiblemente generaron los casos. Se consideró un hospital de alta complejidad como escenario propicio, dada su naturaleza como centro de referencia, donde se atendieran muchas gestantes con patologías complejas, por lo que fuese mandatorio vigilar morbilidad materna extrema - MME (un indicador mucho más conocido).

\section{Metodología}

Se realizó un estudio analítico de corte transversal, retrolectivo, a partir del análisis de las historias clínicas de todos los neonatos con morbilidad neonatal extrema que ingresaron a un hospital de alta complejidad durante el 2013 a través del servicio de urgencias pediátricas o desde la sala de partos.

Dado que la MNE es un evento de vigilancia epidemiológica de baja frecuencia, se hizo un muestreo por casos consecutivos. Fueron incluidos neonatos con cualquiera de las siguientes condiciones (criterios de la Dra. Pileggi \& et al., 2014): cirugía mayor, convulsiones, hemorragia aguda severa que amenazara la vida del paciente, cardiopatía congénita compleja, reanimación neonatal avanzada, peso al nacer menor de 1,000 gramos, Apgar menor de 7 a los 5 minutos, criterios relacionados con falla orgánica (encefalopatía hipóxico-isquémica - EHI Sarnat II o III; gases arteriales con diferencia alvéolo-arterial de oxígeno $>300$; base exceso menor de $-12 \mathrm{mEq} / \mathrm{L}$; índice de oxigenación $>25$, oliguria $<0.5 \mathrm{cc} / \mathrm{Kg} /$ hora por 8 horas), ventilación mecánica por más de 7 días, uso de inotrópicos, uso de antiarrítmicos, requerimiento de cardioversión o necesidad de diálisis (5).

Para identificar los casos se revisaron periódicamente las historias clínicas de los servicios de urgencias pediátricas, neonatología y unidad de cuidado intensivo. Se excluyeron los neonatos en los que no se pudo recolectar la información o que ingresaron muertos al servicio de urgencias de pediatría o fallecieron en sala de partos (óbitos).

Se utilizó el formato diseñado por el grupo que lideró la prueba piloto colombiana, con autorización de parte del Dr. Jorge Mejía (líder nacional del grupo OPS de MNE) (1). Se recolectaron las variables agrupadas en cuatro grupos de datos que permitieron evaluar la morbilidad neonatal extrema de la siguiente manera: (A) información general de la madre y el embarazo (departamento geográfico, fecha del parto, procedencia, área de residencia, edad, nivel educativo, estado civil, ocupación, régimen de afiliación, fórmula obstétrica, periodo intergenésico, controles prenatales, edad gestacional, vía del parto, instrumentación del parto, MME, causa de la MME, otros eventos de MME, supervivencia al egreso); (B) datos del recién nacido (lugar donde ocurrió la MNE, fecha ingreso a unidad neonatal, sexo, edad gestacional por examen físico, Apgar, peso, requerimiento de reanimación, fecha de egreso, supervivencia al egreso); (C) datos relacionados con los criterios de inclusión (mencionados previamente) y el diagnóstico (causa principal de la MNE, otros eventos de MNE, causa perinatal de MNE); y (D) datos relacionados con el análisis cualitativo de la atención neonatal (retrasos tipo I - IV).

Se valoró cada caso en una junta del grupo de investigación una vez a la semana, donde se definió cuál era la causa principal de la MNE, se hallaron otras causas asociadas y se identificaron los retrasos en la atención del neonato y de las madres según la clasificación OPS utilizada dentro del modelo de vigilancia nacional $(1,15)$. Dicha clasificación es la siguiente:

A. Retraso tipo I. Demora para reconocer la necesidad de atención en salud. 
B. Retraso tipo II. Demora asociada con el acceso a la atención de la gestante o del neonato desde el sitio de residencia.

C. Retraso tipo III. Demora en la referencia una vez la gestante o su neonato acceden a los servicios de salud.

D. Retraso tipo IV. Demora en recibir una calidad de atención eficiente en los servicios de salud.

Se describieron las variables por medio del cálculo de medidas de tendencia central y dispersión, o de proporciones y tasas; y se obtuvo la frecuencia de los eventos de MNE.

Dentro del análisis de casos, en la revisión de los retrasos en la atención asociados a la MNE ocurrió un fenómeno de saturación dentro del análisis cualitativo con los primeros 60 sujetos consecutivos, debido a que ocurría el mismo tipo de retraso en la mayoría de los casos; esto conllevaba a que analizar los restantes 60 casos no modificara las conclusiones debido a la gran frecuencia de los retrasos predominantes (21-23). Se hizo exploración de niveles de asociación de los retrasos con respecto a los eventos de MNE y sus desenlaces mediante pruebas de chi cuadrado de Pearson.

El presente estudio se consideró una investigación sin riesgo, dada su naturaleza retrospectiva, según las normas colombianas establecidas en la Resolución 8430 de 1993. Por estos motivos, se dispensó de la necesidad de consentimiento informado por el Comité de Ética en Investigación Científica de la Universidad Industrial de Santander al momento de su aprobación y se obtuvo autorización de la institución hospitalaria que custodiaba la documentación para el acceso a la información. Para garantizar el anonimato se realizó codificación de la base de datos y la información de todos los pacientes se presentó de forma agrupada.

\section{Resultados}

En el 2013 ingresaron 1,190 neonatos a la institución, se hallaron 120 casos de MNE (figura 1). La tasa de MNE encontrada fue de 59 por cada 1,000 nacidos vivos. Se presenta la información de los primeros 60 casos analizados debido al fenómeno de saturación descrito previamente.

Figura 1. Flujograma de captación y seguimiento de los pacientes. Los neonatos muertos del inicio del flujograma son casos donde estaban muertos al inicio de la atención en el Hospital. El análisis posterior se realizó con los primeros 60 casos por saturación de datos acerca de los retrasos neonatales.

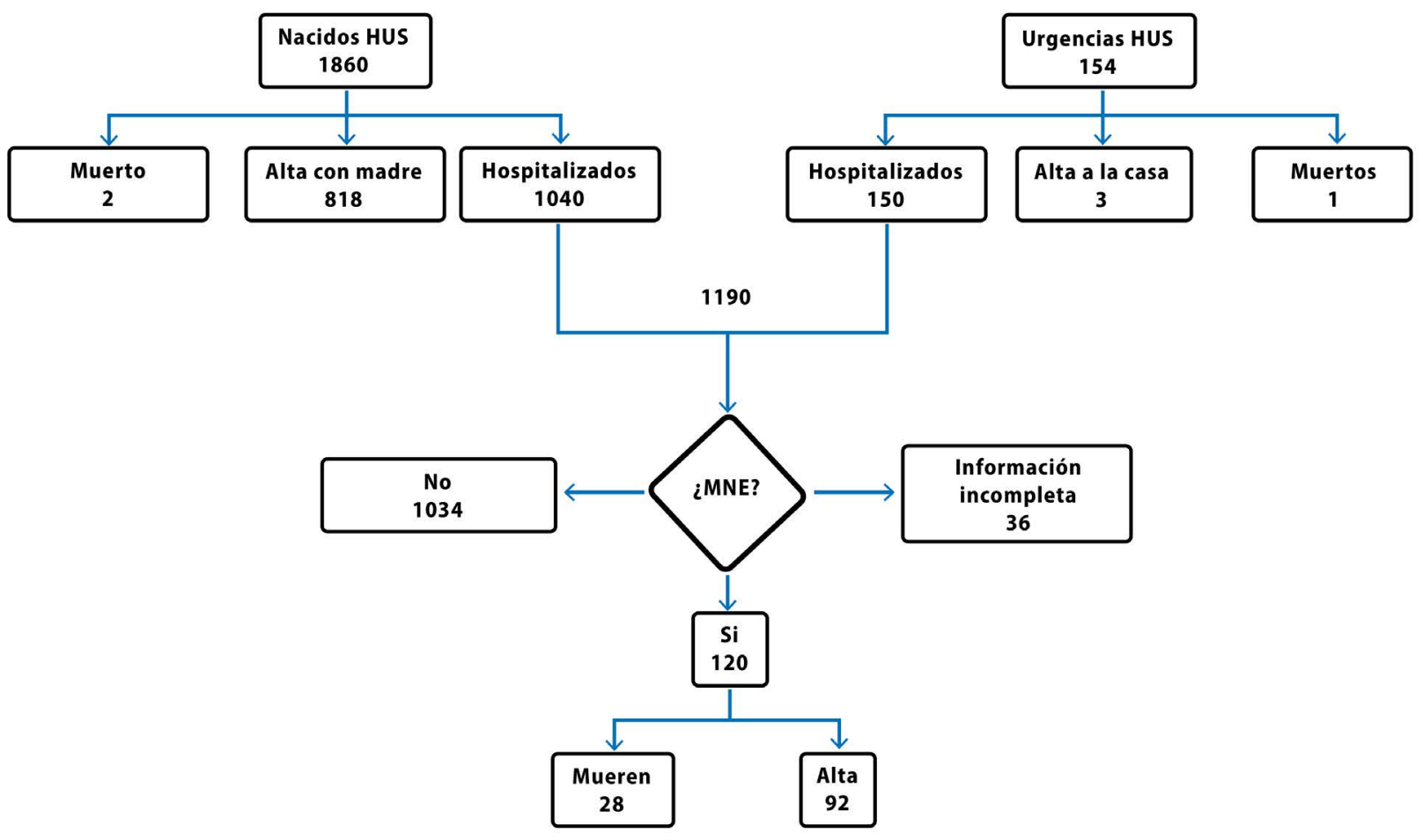

Fuente: elaborada por los autores 
En cuanto a las características de la madres y sus embarazos, se encontró una mediana de 22 años (RIQ 19 - 27 años); todas pertenecían al régimen subsidiado de la seguridad social colombiana, el $60.0 \%$ de maternas fueron multigestantes, con una mediana de 2 gestaciones (RIQ 1 - 3); se documentó un periodo intergenésico menor a 18 meses en el 27.9\%; y hubo control prenatal en el 78.3\% de los casos (media 4 y DS 2), la mayoría desde el ler trimestre (64.0\%). También se observó que había alguna morbilidad en el $63.3 \%$ de las gestantes (tabla 1) y se presentó algún retraso en su atención en el $58.4 \%$, predominando el tipo I $(45.4 \%)$. A su vez, se encontró que, dentro esta categoría, el $44 \%$ correspondía a ausencia o inicio tardío del control prenatal. En el tipo IV se hallaron problemas tanto en el HUS como en las instituciones de procedencia. Estos datos se muestran en la tabla 2.

Tabla 1. Eventos de MME del estudio.

\begin{tabular}{lcc}
\hline \multicolumn{1}{c}{ Causa } & Cantidad & Prevalencia \\
\hline Trabajo de parto pretérmino & 11 & $18.3 \%$ \\
\hline Preeclampsia severa & 8 & $13.3 \%$ \\
\hline $\begin{array}{l}\text { Ruptura prematura de } \\
\text { membranas }\end{array}$ & 3 & $5.0 \%$ \\
\hline Infección de vías urinarias & 2 & $3.3 \%$ \\
\hline Corioamnionitis & 2 & $3.3 \%$ \\
\hline Neumonía & 2 & $3.3 \%$ \\
\hline Oligohidramnios & 1 & $1.7 \%$ \\
\hline Otras causas & 9 & $15.0 \%$ \\
\hline Ninguna & 22 & $36.7 \%$ \\
\hline Total & $\mathbf{6 0}$ & $\mathbf{1 0 0 \%}$
\end{tabular}

Se presentan los eventos más importantes en los 60 casos de MNE analizados. La prematurez fue el evento más relevante.

Fuente: Elaboración propia.

Tabla 2. Retrasos en la atención materna y neonatal de los casos de MNE.

\begin{tabular}{|lcc|}
\multicolumn{1}{r}{ Tipo retraso } & Materno & Neonatal \\
\hline Ninguno & $25(41.7 \%)$ & $28(46.7 \%)$ \\
\hline Tipo I & $20(45.4 \%)$ & $0(0.0 \%)$ \\
\hline Tipo II & $2(4.5 \%)$ & $2(3.3 \%)$ \\
\hline Tipo III & $9(22.7 \%)$ & $13(21.7 \%)$ \\
\hline Tipo IV & $12(27.2 \%)$ & $24(40.0 \%)$
\end{tabular}

Ocurrieron retrasos maternos en 35 casos de MNE (58.4\%) y neonatales en 32 casos $(53.3 \%)$. Hubo casos con más de un tipo de retraso, contándose por separado cada uno. Se reporta el total acumulado de eventos y la frecuencia relativa respecto al total de retrasos. Hay un predominio amplio de los retrasos maternos tipo I y neonatales tipo IV

Fuente: Elaboración propia.
En los neonatos se halló que el $63.3 \%$ de los nacimientos fueron por cesárea, con una mediana de edad gestacional al nacer de 34 semanas (RIQ 28 - 38), y se presentó un $73.2 \%$ de partos pretérminos. Por cesárea nació el $72.7 \%$ de los neonatos prematuros y el $37.5 \%$ de los nacidos a término $(p=0.012)$. Los eventos principales de MNE fueron: prematuros y/o asfixiados (55\%), SDRN (22\%) y malformaciones (12\%), como se muestra en la tabla 3. Dentro de los criterios de MNE se observó (tabla 4): Apgar promedio al minuto de 5 (DS 2 puntos), y a los cinco minutos tuvo una mediana de 7 (RIQ 5 - 9 puntos); el $39.9 \%$ de los niños pesaron menos de $1,500 \mathrm{~g}$ (mediana 2,000 g, RIQ $1,050-2,670$ g) y un $23 \%$ estuvo por debajo de 1,000 g; el 35.0\% de los casos requirió alguna maniobra de reanimación y el $13.3 \%$ falleció. Dentro de los fallecidos, el $75 \%$ de las defunciones correspondieron a neonatos menores de 30 semanas de gestación, y un $87 \%$ de los casos tuvieron algún tipo de retraso en su atención, prevaleciendo el tipo I por ausencia o pobre control prenatal. La tasa de mortalidad neonatal fue de 13.9: 1000 nacidos vivos.

En referencia a la MNE (tabla 4), entre los 150 criterios encontrados en los 60 neonatos hubo predominio (52.0\%) de los criterios de manejo, siendo los principales el requerimiento de reanimación neonatal avanzada, de ventilación mecánica y de inotropía; en los criterios de signos y síntomas predominaron elApgar $<7$ a los 5 minutos $(48.2 \%)$ y el peso $<1000 \mathrm{~g}(25.0 \%)$. Se encontró que cerca de la mitad cumplía 3 o más criterios de MNE (42\%). La mayoría de los casos de MNE (78.3\%) necesitaron manejo en unidad de cuidado intensivo, fueron atendidos en el HUS un 69.9\% de estos niños; sin embargo, hubo 9 casos donde el traslado a la UCIN fue diferido (cinco remisiones externas y cuatro ingresos tardíos). El tiempo de espera fue de 29 horas (RIQ $12-86$ ).

Tabla 3. Eventos principales de MNE.

\begin{tabular}{lcc}
\multicolumn{1}{c}{ Evento } & Cantidad & Prevalencia \\
\hline Prematuridad & 18 & $30.0 \%$ \\
\hline Asfixia neonatal & 15 & $25.0 \%$ \\
\hline $\begin{array}{l}\text { Síndrome de dificultad } \\
\text { respiratoria }\end{array}$ & 13 & $21.6 \%$ \\
\hline Malformación gastrointestinal & 7 & $11.6 \%$ \\
\hline Hipertensión pulmonar & 3 & $5.0 \%$ \\
\hline Convulsiones & 1 & $1.6 \%$ \\
\hline Otras causas & 3 & $5.0 \%$ \\
\hline Total & $\mathbf{6 0}$ & $\mathbf{1 0 0 \%}$
\end{tabular}

Se reporta la causa principal en cada caso, así existiesen otras causas en un caso específico e independiente del total de casos afectados por un mismo evento. Los cuatro más frecuentes se relacionan con los indicadores de la definición operativa abreviada de MNE.

Fuente: Elaboración propia. 
Tabla 4. Criterios de MNE de los casos analizados $(\mathrm{N}=60)$.

\begin{tabular}{|lcc}
\multicolumn{1}{c}{ Criterios } & Frecuencia & $\%$ \\
\hline Signos y síntomas & & \\
\hline Apgar $<7$ a los 5 minutos & 27 & $45.0 \%$ \\
\hline Peso $<1,000 \mathrm{~g}$ & 14 & $23.0 \%$ \\
\hline Hemorragia aguda masiva & 6 & $10.0 \%$ \\
\hline Convulsiones & 5 & $8.3 \%$ \\
\hline $\begin{array}{l}\text { Malformación que requiere cirugía } \\
\text { inmediata }\end{array}$ & 3 & $5.0 \%$ \\
\hline Cardiopatía congénita compleja & 1 & $1.7 \%$ \\
\hline Falla orgánica & & \\
\hline Base exceso $<-12$ & 8 & $13.3 \%$ \\
\hline Delta alvéolo-arterial O2 $>300$ & 2 & $3.3 \%$ \\
\hline Sarnat II-III & 2 & $3.3 \%$ \\
\hline Diuresis $<0.5$ cc/k-hora & 2 & $3.3 \%$ \\
\hline Índice de oxigenación $>25$ & 2 & $3.3 \%$ \\
\hline Manejo & & \\
\hline Uso de inotrópicos & 32 & $53.3 \%$ \\
\hline Ventilación mecánica $\geq 7$ días & 25 & $41.7 \%$ \\
\hline Reanimación neonatal avanzada & 21 & $35.0 \%$ \\
\hline
\end{tabular}

Se presenta la frecuencia absoluta y relativa de cada criterio. Hubo casos con más de un evento.

Fuente: Elaboración propia.

Tabla 5. Análisis bivariado de los retrasos maternos y neonatales respecto del criterio de gravedad (3 o más criterios de MNE). .

\begin{tabular}{|c|c|c|}
\hline Tipo retraso & $\begin{array}{l}\text { Retrasos maternos } \\
\text { en los neonatos } \geq 3 \\
\text { criterios de } \mathrm{MNE}\end{array}$ & $\begin{array}{c}\text { Retrasos en } \\
\text { neonatos con } \geq 3 \\
\text { criterios de } \mathrm{MNE}\end{array}$ \\
\hline Ninguno & $9(15.0 \%)$ & $7(11.6 \%)$ \\
\hline Tipo I & $\begin{array}{c}7(11.6 \%) \\
\mathrm{X}^{2}=9.2000 \\
\mathrm{p}=0.163\end{array}$ & $0(0.0 \%)$ \\
\hline Tipo II & $\begin{array}{c}1(1.6 \%) \\
\mathrm{X}^{2}=39.3103 \\
\mathrm{p}=0.000\end{array}$ & $\begin{array}{c}2(3.3 \%) \\
\mathrm{X}^{2}=39.3103 \\
\mathrm{p}=0.000\end{array}$ \\
\hline Tipo III & $\begin{array}{c}6(10.0 \%) \\
X^{2}=4.6400 \\
p=0.591\end{array}$ & $\begin{array}{c}6(10.0 \%) \\
X^{2}=4.7463 \\
p=0.577\end{array}$ \\
\hline Tipo IV & $\begin{array}{c}8(13.3 \%) \\
X^{2}=12.3611 \\
p=0.054\end{array}$ & $\begin{array}{c}14(23.3 \%) \\
X^{2}=13.6343 \\
p=0.034\end{array}$ \\
\hline
\end{tabular}

Se presentan los valores de chi cuadrado $\left(\mathrm{X}^{2}\right)$ y de $\mathrm{p}$. Sólo hay asociación directa con los retrasos neonatales tipo IV. En los retrasos tipo II la asociación es a la inversa, donde es más probable que no existan cuando hay $\geq 3$ criterios de MNE.

Fuente: Elaboración propia.
Finalmente, con respecto al análisis cualitativo de la atención de los neonatos (tabla 2), se encontró que el $53.3 \%$ presentaron al menos un tipo de retraso, el tipo IV $(40 \%)$ fue el más frecuente. Se halló un número no despreciable de retrasos tipo III (21.7\%). Al discriminar los casos tipo IV por categorías, se encontró que la falta de adherencia a protocolos fue el problema más relevante. También se determinó que 25 de los pacientes (36.7\% Vs. $41.1 \%$ nacional) cumplían 3 o más criterios de MNE (marcador de gravedad), y dentro de estos sólo el $16.6 \%$ no tenían retrasos en la atención de la madre o del neonato (1). Al realizar el análisis bivariado, existió una asociación estadísticamente significativa entre la presencia de $\geq 3$ criterios de MNE y de los retrasos neonatales tipo IV (tabla 5); además, la presencia de convulsiones y el uso de inotrópicos fueron las variables de MNE que se asociaron con los retrasos tipo I y IV, respectivamente $(\mathrm{X} 29.64$ y $12.27, \mathrm{p}=0.01)$.

\section{Discusión}

La MNE guarda una estrecha relación con la MME y sus determinantes (incluyendo los retrasos en la atención), aun así, suelen intervenir otros factores, que incluso son más relevantes. Ejemplo claro en nuestro estudio fue el predominio de retrasos maternos tipo I, por el pobre control prenatal; este es un problema conocido en el mundo y en nuestro país, que suma morbilidad a sus embarazos, pero no explica todos los casos de MNE. Por este motivo, se requieren análisis más amplios para su estudio $(1,20,24,25)$.

Después de hacer una revisión de lo reportado por países de bajos ingresos socioeconómicos (donde la mortalidad neonatal se reduce muy lentamente y las barreras de acceso son mayores), se encuentran una serie de estudios etíopes muy recientes. Yohannes et al. (2019) realizaron un estudio de casos y controles no emparejados (134 casos y 268 controles), una población con tasas de mortalidad superior a la nuestra (29 muertes por cada 1,000 nacidos vivos); encontraron que una distancia mayor a $15 \mathrm{Km}$, embarazo no planeado y no deseado, menos de 4 controles prenatales y parto instrumentado, estuvieron asociados a MNE $(26,27)$. Otro estudio similar conducido por Mersha et al. (2019), con 121 casos y 363 controles, mostró asociación en común con el control prenatal deficiente y el parto instrumentado, aunque identificó asociación con otros factores (multiparidad, ruptura prematura de membranas y cesáreas) (28). El estudio de corte transversal retrolectivo de Tassew et al. (2020), con 422 neonatos y $32.2 \%$ de casos de MNE, mostró asociación similar con controles prenatales deficientes, procedencia 
lejana y parto instrumentado; estos mismos autores también reportaron importancia de otros factores como ser primigestantes, hipertensión asociada al embarazo y hemorragia anteparto (eventos de MME) (29). Gebrehana et al. (2020), también realizaron un estudio de corte transversal, con 404 neonatos y $23.3 \%$ de casos de MNE, encontrando asociación similar con datos clínicos (parto instrumentado, primigestantes y ruptura prematura de membranas); reportaron asociación con casos remitidos de otras instituciones (30). Por último, un estudio de cohorte realizado por Tekelab et al. (2020), con 2,704 nacidos vivos y 122 casos de MNE, reportó una tasa de MNE de 45 casos por cada 1,000 nacidos vivos y mostró asociación con un nivel socioeconómico bajo, periodos intergenésicos cortos y la MME (que suele estar directamente relacionada con el control prenatal) (31).

En la categoría de países de ingresos medianos bajos, se encontró un reporte indio reciente por Sunny et al. (2020), de corte transversal, en un hospital de alta complejidad, con 210 neonatos y una tasa de MNE muy alta en 75 casos por cada 1,000 nacidos vivos. Los factores asociados fueron pobre control prenatal (sin ecografías), anemia materna, ruptura prematura de membranas, oligohidramnios y parto instrumentado (32).

En países latinoamericanos con ingresos similares al nuestro (medianos altos y/o altos), los estudios más recientes son los brasileros (también fueron los pioneros en hablar de MNE, como se mencionó previamente). Resalta el estudio hecho por Ximenes et al. (2020), donde informan datos de una primera observación en 2012 (tiempo similar al presente estudio) y una nueva en 2016, con la misma metodología. En el 2012 encontraron 302 casos más severos, con bajo peso al nacer y sin controles prenatales; y en el 2016 reportaron 243 casos, con más admisiones a cuidado intensivo. La tasa de MNE fue de 145 y de 99 casos por 1,000 nacidos vivos, respectivamente (asociada a que fueron casos reportados en un hospital de alta complejidad). Este estudio particularmente usa una definición abreviada de MNE donde sólo se toman en cuenta cuatro factores por su sencillez (información obtenible desde bases de datos generales): edad gestacional $<33$ semanas, peso al nacer $<1,750 \mathrm{~g}$, Apgar 5 minutos $<7$ y admisión a la UCI neonatal.

Resaltan que el modelo está orientado a predecir muertes neonatales, sin tener en cuenta la calidad de la atención, por lo que requiere complementarse si se va a utilizar con este fin $(33,34)$. En concordancia, una revisión previa por la Dra. Costa Carvalho et al. en el 2019 mostró que los criterios de MNE aún siguen evolucionando con variaciones en el número de criterios y los cortes para el peso y la edad gestacional (35). Estudios como el de Barbosa et al. en el 2018, siguiendo una cohorte con 221 casos de MNE en un hospital de alta complejidad al norte de Brasil, con una tasa de MNE de 220 casos por cada 1,000 nacidos vivos y asociación principal hacia controles neonatales insuficientes y malformaciones fetales, donde se utilizaron los criterios originales de la Dra. Pileggi, reflejan que estos criterios siguen vigentes (36). Es llamativo que estudios más antiguos de este grupo de países reportan tasas de MNE mucho menores $(17$ - 33 casos por cada 1,000 nacidos vivos); esta situación se puede explicar con base en que fueron datos de varios centros y con mayor número de neonatos, sin variaciones significativas en la mortalidad $(37,38)$.

En países de altos ingresos hay datos agrupados obtenibles del estudio de la Dra. Pileggi et al. en 2014, quienes analizaron 290,610 neonatos de la encuesta global de salud materna y perinatal y 310436 neonatos vivos de la encuesta internacional de salud materna y neonatal, reportando una tasa de MNE de 72.5 casos por cada 1,000 nacidos vivos y los siguientes estadísticos de MNE para muerte neonatal temprana: sensibilidad $92.8 \%$, especificidad $92.7 \%$, valor predictivo positivo 12.7 y valor predictivo negativo 0.08 (5). En Rusia, con datos más recientes reportados por Bushtyrev et al. en 2016 (analizaron 16,766 neonatos en un centro de alta complejidad), reportaron una tasa de MNE de 85.5 por cada 1,000 nacidos vivos (39).

Un dato importante al comparar las tasas de MNE, es que en los países con ingresos económicos bajos hay una mayor mortalidad neonatal (13). En países con ingresos medianos y altos hay menor mortalidad, pero la MNE suele ser mayor (8). Lo anterior parece corresponder con un mayor éxito de los recursos y las intervenciones médicas en evitar las muertes, pero no la morbilidad.

En Colombia sigue siendo vigente hablar de MNE dado que el contexto de la mortalidad neonatal no ha cambiado significativamente según los datos reportados por Rojas et al. entre 2008 - 2017, con mayores tasas de mortalidad neonatal condicionadas por grandes distancias geográficas y el bajo peso al nacer (18); por este motivo, sigue siendo deseable implementar estrategias de vigilancia e intervención nuevas, como la que se viene proponiendo desde el 2014 y que se aplica en el presente estudio (1). Desde la realización del presente estudio en 2013, solo se ha publicado un estudio sobre factores asociados a mortalidad neonatal por Mendoza et al. en 2017, con datos de 10 años 
previos y análisis de 125 muertes neonatales, donde los determinantes fueron los mismos que se asocian con MNE (40).

En relación con los hallazgos del presente estudio, la tasa de MNE fue cuatro veces mayor que la mortalidad, por lo que el grupo poblacional a intervenir siempre será mayor, lo que dimensiona mejor el impacto del problema de una atención deficiente en salud. Esta situación es conocida en la comunidad médica (8). Los valores fueron comparables con los reportados para países de ingresos medianos y altos, indicando que los retos en salud pública probablemente son similares a los de toda Latinoamérica.

Por otro lado, haber encontrado en este estudio una preponderancia del peso $<1,500 \mathrm{~g}$, ventilación mecánica, Apgar 5 minutos $<7$ y malformaciones congénitas, entre las demás variables de MNE, es acorde con lo informado en instituciones de alta complejidad a nivel mundial, relacionado en los párrafos previos. Es de anotar que se ha propuesto que la edad gestacional $<33$ semanas y sólo estos cuatro indicadores sean considerados como su definición operativa $(34,38)$. Adicionalmente, es necesario recordar que todos los hospitales incluidos en la prueba piloto colombiana fueron de alta complejidad, lo que hace esperable que la falta de recursos humanos y técnicos, junto con la poca adherencia a protocolos de manejo, contribuyesen a que ocurrieran los retrasos tipo IV, tanto locales como nacionales (1). El hecho que los neonatos que con mayor frecuencia tienen desenlace fatal sean los prematuros extremos y de bajo peso, indica que priorizar estos casos es mandatorio en políticas respecto de la MNE.

Hablando propiamente sobre el proceso de análisis de $\mathrm{MNE}$, se requiere un grupo interdisciplinario para su ejecución y se sugiere direccionar la información a un centro de recolección nacional. A su vez, la formación continuada del personal parece ser la mejor opción para disminuir la probabilidad de acciones inseguras.

\section{Conclusiones}

La metodología de evaluación de la MNE es una herramienta valiosa de vigilancia en salud pública, conocida en el mundo desde el 2007 y en Colombia desde el 2014. Este es el primer reporte científico de su uso en el país. Se encontró una tasa de MNE similar a otros países con los mismos ingresos económicos. Los eventos más relevantes de MNE (peso $<1,500 \mathrm{~g}$, ventilación mecánica, Apgar 5 minutos $<7$ y malformaciones congénitas) fueron los más observados en el estudio. Los retrasos en la atención más frecuentes fueron los maternos tipo I y los neonatales tipo III y IV. Se recomienda hacer análisis interdisciplinario de estos casos y destinar recursos a la educación continuada del personal de salud que atiende neonatos.

\section{Conflicto de intereses}

Los autores declaran no tener conflicto de intereses. El estudio fue financiado por la Universidad Industrial de Santander y con recursos propios de los autores. La institución hospitalaria no aportó recursos para su ejecución y no tuvo participación en el análisis de los retrasos en la atención.

\section{Referencias}

1. Ortíz Lizcano EI, Mejía López J, Acuña JM, Quintero J $\mathrm{CA}$, Jiménez $\mathrm{C}$, Medina $\mathrm{C}$, et al. Modelo de vigilancia de la morbilidad neonatal extrema y primer análisis agregado de casos. Fondo de Población de las Naciones Unidas (UNFPA), editor. Bogotá; 2014. 1-41 p.

2. Pileggi C, Souza JP, Cecatti JG, Faundes A. Neonatal near miss approach in the 2005 WHO Global Survey Brazil. J Pediatr (Rio J) [Internet]. 2010;86(1):21-6. https://doi.org/10.1590/S0021-75572010000100005

3. Organización Mundial de la Salud-OMS-. Reducción de la Mortalidad y Morbilidad Neonatal en América Latina y el Caribe. 1st ed. Serviprensa, editor. Guatemala: OMS; 2007. 41 p.

4. Avenant T. Neonatal near miss: a measure of the quality of obstetric care. Best Pract Res Clin Obstet Gynaecol [Internet]. 2009;23(3):369-74. https://doi. org/10.1016/j.bpobgyn.2008.12.005

5. Pileggi-Castro C, Camelo JS, Perdoná GC, MussiPinhata MM, Cecatti JG, Mori R, et al. Development of criteria for identifying neonatal near-miss cases: analysis of two WHO multicountry cross-sectional studies. BJOG. 2014;121 Suppl:110-8. https://doi. org/10.1111/1471-0528.12637

6. Santos JP, Pileggi-Castro C, Camelo JS, Silva AA, Duran P, Serruya SJ, et al. Neonatal near miss: A systematic review. BMC Pregnancy Childbirth. 2015;15(1):1-10. https://doi.org/10.1186/s12884-015-0758-y

7. Santos J, Cecatti J, Serruya S, Almeida P, Duran P, Mucio B, et al. Neonatal Near Miss: the need for a standard definition and appropriate criteria and the rationale for a prospective surveillance system. Clinics [Internet]. 2015;70(12):820-6. https://doi. org/10.6061/clinics/2015(12)10

8. Surve S, Chauhan S, Kulkarni R. Neonatal near miss review: Tracking its conceptual evolution and way forward. Curr Pediatr Res. 2017;21(2):264-71. 
9. Cáceres Manrique $\mathrm{F}$ de $\mathrm{M}$, Meza Durán EE, Arias Barrera CI. Factores de riesgo asociados a la mortalidad neonatal. Hospital Universitario Ramón González Valencia, 2000. MedUNAB. 2001;4(11):112-20 Disponible en: https://revistas. unab.edu.co/index.php/medunab/article/view/310

10. Delgado M, Muñoz A, Orejuela L, Sierra $\mathrm{CH}$. Algunos factores de riesgo para mortalidad neonatal en un hospital de III nivel, Popayán. Colomb Med. 2003;34(4):179-85.

11. Barrios V, Rodríguez C, Sánchez R. Factores de riesgo asociados a mortalidad neonatal en unidades de cuidados intensivos neonatales. Estudio multicéntrico. Barranquilla, enero de 2012-mayo de 2013. Biociencias. 2013;8(1):45-52.

12. Díaz Martínez LA, Cáceres Manrique $\mathrm{F}$ de $\mathrm{M}$, Becerra Mojica $\mathrm{CH}$, Vera LA, Uscátegui Dacartt AM. Causas y determinantes de la mortalidad perinatal, Bucaramanga, 1999. Vol. 3, MedUNAB. 2000. p. 5-16. Disponible en: https://revistas.unab. edu.co/index.php/medunab/article/view/344

13. Wang H, Liddell CA, Coates MM, Mooney MD, Levitz CE, Schumacher AE, et al. Global, regional, and national levels of neonatal, infant, and under-5 mortality during 1990-2013: a systematic analysis for the Global Burden of Disease Study 2013. Lancet. 2014;384(9947):957-79. https://doi. org/10.1016/S0140-6736(14)60497-9

14. Equipo maternidad segura - subdirección prevención vigilancia y control en salud pública. Mortalidad perinatal y neonatal tardía [Internet]. 2016. (Protocolo de Vigilancia en Salud Pública). Disponible en: http://www.hosusana.gov.co/sites/ default/files/u1/capacitacion/PRO Mortalidad perinatal y neonatal(1).pdf

15. Thaddeus S, Maine D. Too far to walk: Maternal mortality in context. Soc Sci Med [Internet]. 1994 Apr;38(8):1091-110. https://doi. org/10.1016/0277-9536(94)90226-7

16. Moura PMSS, Maestá I, Rugolo LMSS, Angulski LFRB, Caldeira AP, Peraçoli JC, et al. Risk factors for perinatal death in two different levels of care: A case-control study. Reprod Health. 2014;11(1):1-7. https://doi.org/10.1186/1742-4755-11-11

17. Ministerio de Salud y Protección Social. PDSP, 2012-2021. Bogotá, Colombia; 2013.

18. Rojas-Gualdrón DF, Velásquez-Ospina J, TejadaTobon M, Pineda-SánchezJ.Distribución geográfica del cambio en mortalidad neonatal según bajo peso al nacer, Colombia 2008-2017. Rev la Univ Ind Santander Salud. 2020;52(2):119-28. https://doi. org/10.18273/revsal.v52n2-2020006

19. Rojas-Gualdrón DF, Fernando, Caicedo-Velásquez B. Geographical Accessibility to Obstetric and Neonatal
Care and its Effect on Early Neonatal Mortality in Colombia , 2012-2014. MedUNAB . 2017;20(1):718. https://doi.org/10.29375/01237047.2670

20. Costa Carvalho OM, Viana Junior AB, Costa Carvalho AM, Madeiro Leite ÁJ, Arrais Nobre R, Alencar Costa Bessa OA, et al. Delays in obstetric care increase the risk of neonatal near-miss morbidity events and death: A case-control study. BMC Pregnancy Childbirth. 2020;20(1):1-10. https://doi. org/10.1186/s12884-020-03128-y

21. Morse JM. Emerger los datos: los procesos cognitivos del análisis en la investigación cualitativa. 1st ed. Universidad de Antioquia, editor. Asuntos críticos en los métodos de investigación cualitativa. Medellín; 2003. 29-52 p.

22. Martínez-Salgado C. El muestreo en investigación cualitativa. Principios básicos y algunas controversias. Cienc e Saude Coletiva. 2012;17(3):613-9. https:// doi.org/10.1590/S1413-81232012000300006

23. Schettini P, Cortazzo I. Análisis de datos cualitativos en la investigación social. 1st ed. Dirección de Comunicación Visual de la UNLP, editor. La Plata - Buenos Aires, Argentina: Editorial de la Universidad de La Plata; 2015. 120 p. https://doi. org/10.35537/10915/49017

24. Nakimuli A, Mbalinda SN, Nabirye RC, Kakaire O, Nakubulwa S, Osinde MO, et al. Still births, neonatal deaths and neonatal near miss cases attributable to severe obstetric complications: A prospective cohort study in two referral hospitals in Uganda. BMC Pediatr [Internet]. 2015;15(1):1-8. https://doi. org/10.1186/s12887-015-0362-3

25. Acelas-Granados DF, Orostegui A, Alarcón-Nivia MÁ. Factores de riesgo para morbilidad materna extrema en gestantes sin demora en la atención médica según la estrategia camino para la supervivencia. Rev Chil Obstet Ginecol. 2016;81(3):181-8. https:// doi.org/10.4067/S0717-75262016000300003

26. Yohannes E, Assefa N, Dessie Y. Determinants of neonatal near miss among neonates admitted to Ambo University Referral Hospital and Ambo General Hospital, Ethiopia, 2019. Clin J Obstet Gynecol [Internet]. 2020 Jun 24;3:46-53. Disponible en: https://www.heighpubs.org/cjog/cjog-aid1050. php

27. Yohannes E, Assefa N, Dessie Y. Determinants of Neonatal Near Miss among Neonates Admitted to Ambo University Referral Hospital and Ambo General Hospital, Ethiopia, 2019. J Preg Child Heal. 2020;7(1):1-7. Disponible en: https://www. omicsonline.org/peer-reviewed/determinants-ofneonatal-near-miss-among-neonates-admittedto-ambo-university-referral-hospital-and-ambogeneral-hospital-ethiopia-111402.html 
28. Mersha A, Bante A, Shibiru S. Factors associated with neonatal near-miss in selected hospitals of Gamo and Gofa zones, southern Ethiopia: Nested case-control study. BMC Pregnancy Childbirth. 2019;19(1):1-8. https://doi.org/10.1186/s12884019-2684-X

29. Tassew HA, Kassie FY, Mihret MS. Neonatal near Miss and Its Predictors among Neonates Delivered at Debretabor General Hospital, Northern Ethiopia; A Retrospective Analysis. Int J Pediatr. 2020;2020(Mlic):1-8. https://doi. org/10.1155/2020/1092479

30. Gebrehana Belay H, Limenih SK, Wassie TH, Ambie MB. Neonatal Near Miss and Its Associated Factors at Injibara General Hospital, Awi Zone, Northwest Ethiopia, 2019. Explor Res Hypothesis Med. 2020;000(000):1-8. https://doi.org/10.14218/ ERHM.2020.00011

31. Tekelab T, Chojenta C, Smith R, Loxton D. Incidence and determinants of neonatal near miss in south Ethiopia: A prospective cohort study. BMC Pregnancy Childbirth. 2020;20(1):1-13. https://doi.org/10.1186/ s12884-020-03049-w

32. Sunny S, Johnson AR, Thimmaiah S, Suman Rao $\mathrm{PN}$. Incidence and factors associated with early neonatal near miss in a tertiary care hospital in Bengaluru. Indian J Child Health [Internet]. 2020 Sep 25;7(9):380-4. https://doi.org/10.32677/IJCH.2020. v07.i09.006

33. Ximenes de França K, Rodrigues Vilela MB, Germano de Frias P, Wanick Sarinho S. EARLY NEONATAL NEAR MISS IN A UNIVERSITY HOSPITAL: COMPARATIVE CROSS-SECTIONAL STUDY. Rev Paul Pediatr [Internet]. 2020;39(e2019317). https://doi.org/10.1590/1984-0462/2021/39/2019317

34. Ximenes de França K, Rodrigues Vilela MB, Germano de Frias P, da Silveira Gaspar G, Wanick Sarinho S. Early neonatal near miss identified throug health information systems. Cad Saude Publica. 2018;34(9):1-10.
35. Costa Carvalho OM, de Oliveira Xavier AT, Mendes Gouveia AP, Carvalho Augusto MC, Costa Carvalho FH. Identificação de casos de near miss neonatal: que critérios são usados no cenário brasileiro - revisão integrativa. Rev Med da UFC. 2019;59(3):53-9. $\quad$ https://doi.org/10.20513/24476595.2019v59n3p53-59

36. De Lima THB, Katz L, Kassar SB, Amorim MM. Neonatal near miss determinants at a maternity hospital for high-risk pregnancy in Northeastern Brazil: A prospective study. BMC Pregnancy Childbirth. 2018;18(1):1-8. https://doi.org/10.1186/ s12884-018-2020-X

37. Kale PL, Mello-Jorge MHP de, Silva KS da, Fonseca SC. Neonatal near miss and mortality: factors associated with life-threatening conditions in newborns at six public maternity hospitals in Southeast Brazil. Cad Saude Publica [Internet]. 2017;33(4):112. https://doi.org/10.1590/0102-311x00179115

38. Silva GA, Rosa KA, Saguier ESF, Henning E, Mucha F, Franco SC. A populational based study on the prevalence of neonatal near miss in a city located in the south of Brazil: Prevalence and associated factors. Rev Bras Saude Matern Infant. 2017;17(1):159-67. https://doi.org/10.1590/1806-93042017000100009

39. Bushtyrev BVA, Bushtyreva BIO, Kuznetsova KNB, Budnik BES. Audit of neonatal near miss: Possibilities of improving in perinatology polymorphisms. Akush Ginekol (Sofiia) [Internet]. 2016 Jul 27;7:79-82. https://doi.org/10.18565/aig.2016.7.79-82

40. Mendoza Tascón LA, Gómez Giraldo D, Gómez Giraldo D, Osorio Ruiz MÁ, Villamarín Betancourth EA, Arias Guatibonza MD. Determinantes biológicos de mortalidad neonatal, en una población de mujeres adolescentes y adultas de un hospital en Colombia. Rev Chil Obstet Ginecol [Internet]. 2017;82(4):424-37. https://doi.org/10.4067/s0717-75262017000400424 